\title{
Abnormal wound healing responses in pulmonary fibrosis: focus on coagulation signalling
}

\author{
R.C. Chambers
}

ABSTRACT: The normal response of tissue to injury involves a sequence of overlapping events, which need to occur in a timely and controlled manner for successful tissue repair and restoration of normal function. Failure to control the healing process can lead to considerable tissue remodelling and the replacement of functional tissue with permanent fibrous scar tissue.

It is proposed that pulmonary fibrosis arises from repetitive, widespread epithelial injury. However, the nature of the insult for the most common and most fatal form of pulmonary fibrosis, idiopathic pulmonary fibrosis (IPF), is currently unknown and the pathogenetic pathways leading to IPF remain to be fully elucidated. Increasing evidence suggests that abnormalities in a number of pathways involved in the wound healing response may play central roles.

The present article will briefly review the pathways involved in wound healing focusing on the control of fibroblast/myofibroblast function and the coagulation cascade acting via the family of signalling receptors, the proteinase activated receptors, which influence a range of cellular responses implicated in the development of pulmonary fibrosis.

Understanding the involvement of these pathways in the aberrant wound repair-response in pulmonary fibrosis may lead to the identification of new targets and strategies for therapeutic intervention.

KEYWORDS: Coagulation cascade, lung, myofibroblasts, proteinase-activated receptor

$\mathbf{T}$ he normal response of tissue to injury involves the initiation of a highly coordinated wound repair-programme aimed at restoring normal tissue function. Some of the earliest responses involve the activation of the coagulation cascade, inflammatory cell recruitment and the formation of a provisional matrix to prevent blood loss, infection and promote subsequent wound healing responses. Successful wound repair and the re-establishment of an intact epithelium requires spatially and temporally regulated epithelial and mesenchymal cell responses. Epithelial cell proliferation, migration and differentiation must be coordinated with mesenchymal cell recruitment, proliferation, differentiation with extracellular matrix (ECM) remodelling, and subsequent apoptosis of myofibroblasts [1]. Dysregulation of this orchestrated wound repairresponse can result in pathological scar formation and excessive deposition of collagen and other ECM proteins.

The pathogenetic mechanisms leading to pulmonary fibrosis and, in particular, idiopathic pulmonary fibrosis (IPF) remain poorly understood. However, current evidence suggests that IPF arises as a consequence of chronic, widespread epithelial injury leading to the development of the classical picture of patchy interstitial fibrosis alternating with areas of mild inflammation and normal lung. The failure of classical antiinflammatory and immunosuppressive therapy to impact on the course of this condition supports the notion that IPF results from chronic epithelial injury and dysregulated or inappropriate repair rather than chronic inflammation. The distinctive presence of fibroblastic foci and excessive ECM deposition are thought to lead to the characteristic thickening of alveolar septae and the collapse of normal lung architecture (honeycombing). However, the role of inflammation in driving fibrosis remains at the centre of an interesting debate. The present article will discuss some of the potential mechanisms controlling fibroblast function, with a particular emphasis on coagulation signalling pathways, and what implications this may have for future therapy.
CORRESPONDENCE

R.C. Chambers

Centre for Respiratory Research University College London

Rayne Institute

5 University Street

London WC1E 6JJ

UK

Fax: 442076796973

E-mail: r.chambers@ucl.ac.uk

SUPPORT STATEMENT

R.C. Chambers has received funding from the Wellcome Trust, Medical Research Council, British Lung

Foundation and the Rosetrees Trust (all London, UK).

STATEMENT OF INTEREST

None declared.

European Respiratory Review Print ISSN 0905-9180

Online ISSN 1600-0617 


\section{THE ROLE OF THE MYOFIBROBLAST IN PULMONARY FIBROSIS}

Myofibroblasts are the key effector cells in pulmonary fibrosis, responsible for producing the bulk of ECM proteins deposited within the pulmonary interstitium, including collagens I and III, fibronectin and a number of proteoglycans. These cells are characterised by the de novo expression of $\alpha$-smooth muscle actin ( $\alpha$-SMA) which is organised into functional stress fibres and confers contractile properties [2]. Current evidence suggests that at least three local events are needed to generate $\alpha$-SMA-positive differentiated myofibroblasts: 1) accumulation of biologically active transforming growth factor (TGF)- $\beta 1 ; 2$ ) the presence of specialised ECM proteins, such as the extradomain-A fibronectin (ED-A) splice variant of fibronectin; and 3) high-extracellular stress, arising from the mechanical properties of the ECM and cell remodelling activity [3].

Highly activated myofibroblasts can produce around 3.5 million molecules of collagen per cell per day. These cells are also capable of synthesising numerous inflammatory and fibrogenic mediators as well as several matrix metalloproteases (MMPs) and, thus, play a central role in remodelling the provisional ECM during normal wound healing (fig. 1) [2]. The fate of myofibroblasts in injured tissues is considered to be a critical determinant of whether normal healing occurs or progression to end-stage fibrosis ensues. In normal wound healing, resolution with myofibroblast apoptosis terminates progression. However, in various fibrotic conditions, including pulmonary fibrosis, persistence of TGF- $\beta 1$ expression and ECM deposition promote a pro-survival/anti-apoptotic phenotype so that these cells persist to propagate fibrosis [3].

Current opinion suggests that myofibroblasts may be derived from three potential cellular sources: resident fibroblasts; epithelial cells undergoing epithelial-mesenchymal transition (EMT); and recruited fibrocytes $[2,3]$. The first suggestion is the original and most widely accepted theory and proposes that myofibroblasts are derived from the expansion and

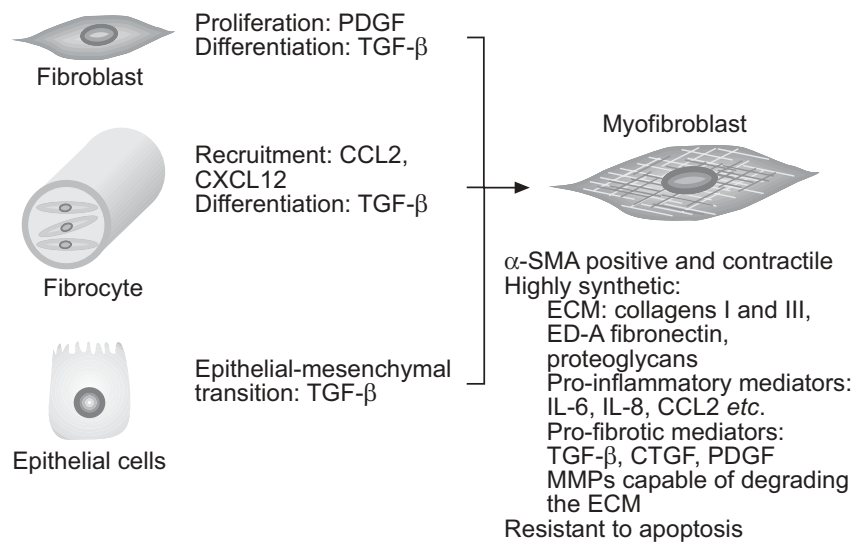

FIGURE 1. The primary effector cell in pulmonary fibrosis is the myofibroblast a cell that is highly synthetic, exhibits a contractile phenotype, and is characterised by the presence of $\alpha$-smooth muscle actin stress fibres. PDGF: platelet-derived growth factor; TGF: transforming growth factor; CCL2: CC motif chemokine ligand 2; CXCL12: CXC motif chemokine ligand 12; $\alpha$-SMA: $\alpha$-smooth-muscle actin; ECM extracellular matrix; ED-A: extra-domain-A fibronectin; IL: interleukin; CTGF: connective tissue growth factor. differentiation of local resident lung fibroblasts under the influence of numerous fibrogenic mediators. These mediators include the mitogen platelet-derived growth factor (PDGF) and the fibrogenic mediator TGF- $\beta 1$, released by the activated epithelium and recruited inflammatory cells (table 1).

The second theory proposes that myofibroblasts are derived from fibrocytes, a distinct population of blood-borne cells that display a unique cell surface phenotype (collagen I+/CD11b+/ CD13+/CD34+/CD45RO+/major histocompatability complex class II+/CD86+) and exhibit potent immunostimulatory activities [4]. The first evidence to show circulating fibrocytes contribute to the pathogenesis of pulmonary fibrosis was observed in a murine model of bleomycin-induced pulmonary fibrosis [5]. In this model, blockade of fibrocyte recruitment by neutralising anti-CXC-motif chemokine ligand (CXCL)12 antibody therapy was protective. Recently, an immunohistochemical study of lung biopsy tissue from patients provided additional support for the role of circulating fibrocytes (most likely recruited through the CXC chemokine receptor 4/ CXCL12 axis) in the expansion of the fibroblast/myofibroblast population in IPF [6]. However, whether recruited fibrocytes are capable of differentiating into fully activated myofibroblasts and are able to produce significant amounts of collagen remains an unresolved issue.

The final theory proposes that myofibroblasts are derived from alveolar epithelial cells undergoing EMT, a process whereby epithelial cells lose their characteristic markers, such as Ecadherin and Zona-occludens-1, and acquire mesenchymal makers such as fibroblast specific protein- 1 and $\alpha$-SMA. The concept of EMT was originally described by embryologists $>20$ yrs ago and occurs in many developmental processes. However, there is emerging evidence that this pathway may be inappropriately re-activated and may contribute to the expansion of the myofibroblast pool in fibrotic lung disease. Alveolar epithelial cells have been shown to undergo EMT after prolonged TGF- $\beta$ exposure in vitro and in vivo $[7,8]$. Evidence for this pathway in patients was provided by biopsy studies demonstrating that IPF lung cells co-express epithelial and mesenchymal markers [7], although this was not a universal finding [9].

\section{THE COAGULATION CASCADE IN PULMONARY FIBROSIS}

The activation of the coagulation cascade is one of the earliest events initiated following tissue injury. It is now well-accepted that the role of the coagulation cascade extends beyond its role in haemostasis and that coagulation proteinases influence a number of cellular responses which are central to wound healing. If coagulation signalling is dysregulated, these cellular responses may then contribute to the tissue fibrosis. In the remaining sections, the present article will focus on the role of coagulation signalling in the context of pulmonary fibrosis.

The primary function of the coagulation cascade is to generate insoluble, cross-linked fibrin strands that bind and stabilise weak platelet haemostatic plugs, which are formed at sites of tissue injury. The formation of this provisional clot is critically dependent on the action of thrombin, and is generated following the stepwise activation of coagulation proteinases via the extrinsic and intrinsic systems [10]. 


\begin{tabular}{lc} 
TABLE 1 & Major positive mediators of lung fibrosis \\
Mediator & Cellular response \\
\hline CCL2 & Mediates fibrocyte recruitment and promotes \\
collagen synthesis via TGF- $\beta$ & Mediates fibrocyte recruitment \\
CCL3 & Regulation of collagen synthesis \\
CCL7 & Fibroblast mitogen and differentiation; \\
CTGF & promotes TGF- $\beta$-dependent ECM synthesis \\
CXCL12 & Fibrocyte recruitment \\
ET-1 & Fibroblast mitogen and chemoattractant, regulation of \\
FGF2 & ECM synthesis and contraction; promotes EMT \\
IGF-1 & Fibroblast mitogen \\
IL-4 & Fibroblast mitogen and promotes collagen synthesis \\
IL-6 & Regulation of collagen synthesis \\
IL-12 & Regulator of $\alpha$-SMA expression in myofibroblasts \\
IL-13 & Regulation of collagen synthesis \\
IL-17 & Induction of TGF- $\beta$ \\
PDGF & Fibroblast proliferation \\
TGF- $\beta$ & Fibroblast mitogen and chemoattractant \\
Thrombin & ECM production, fibroblast proliferation and \\
differentiation; promotes EMT
\end{tabular}

CCL: CC motif chemokine ligand; CTGF: connective tissue growth factor; CXCL12: CXC motif chemokine ligand 12; ET: endothelin; FGF: fibroblast growth factor; IGF: insulin-like growth factor; IL: interleukin; PDGF: platelet-derived growth factor; TGF: transforming growth factor; ECM: extracellular matrix; EMT: epithelial-mesenchymal transition; $\alpha$-SMA: $\alpha$-smooth-muscle actin.

There is good evidence that a tissue factor-dependent extrinsic coagulation pathway is the predominant mechanism by which the coagulation cascade is locally activated in the lungs of patients with pulmonary fibrosis (fig. 2). Tissue factor (TF) is highly upregulated on type-II pneumocytes, and to some extent on alveolar macrophages, in close association with fibrin deposits in the lungs of patients with IPF [11]. TF initiates the coagulation cascade by forming a complex with factor VIIa (FVIIa) complex. The TF-FVIIa complex subsequently catalyses the initial activation of FX to FXa and FIX to FIXa. FXa, in association with activated factor $\mathrm{V}$ (FVa), catalyses the conversion of prothrombin to thrombin, which in turn converts fibrinogen to fibrin (fig. 2). TF-positive cells are found overlying fibrotic areas, suggesting that local TF expression and extravascular activation of the coagulation cascade is closely related to fibrin deposition in IPF (fig. 2) [11-14].

The coagulation cascade is tightly controlled by negativefeedback mechanisms, as well as by circulating and locally produced endogenous anticoagulants. The extrinsic pathway is mainly controlled by TF pathway inhibitor (TFPI) which inactivates TF-FVIIa complexes after binding to FXa. The intrinsic pathway is controlled by antithrombin which inhibits thrombin and other serine proteinases. Another important control mechanism involves the binding of thrombin to the endothelial cell surface receptor, thrombomodulin. During this process, thrombin is converted from a pro-coagulant into an anticoagulant by activating protein $\mathrm{C}$. Protein $\mathrm{C}$ activation is further enhanced by the endothelial cell surface receptor, endothelial cell protein $C$ receptor. Activated protein $C$, in conjunction with protein $\mathrm{S}$, inactivates FVa and FVIIIa and thereby suppresses further thrombin generation. There is good evidence that in patients with pulmonary fibrosis, the balance is greatly shifted in favour of pro-coagulant activity and also antifibrinolytic activity [12].

The main effector enzyme in the fibrinolytic system is plasmin, which is derived from plasminogen via the action of urokinasetype plasminogen activator and tissue-type plasminogen activator, produced by endothelial cells, alveolar epithelial cells and macrophages. Studies in patients with pulmonary fibrosis have shown that inhibitors of this pathway, including plasminogen activator inhibitor-1 (PAI1) and PAI2, protein C inhibitor and thrombin-activatable fibrinolysis inhibitor are increased in the lungs of patients with pulmonary fibrosis [12, 15]. Decreased protein $C$ activation was further found to be associated with abnormal collagen turnover in the intraalveolar space in patients with interstitial lung disease [16].

Evidence that the coagulation cascade may be of pathophysiological significance has come from in vivo models of bleomycininduced pulmonary fibrosis. Targeting of the coagulation cascade in these models, using a variety of strategies such as direct thrombin inhibition [17], TFPI [18], intratracheal administration of activated protein C [19] and aerosolised heparin [20], led to a reduction in lung collagen accumulation and the development of fibrotic lesions. Fibrin acts as a provisional matrix for the recruitment of inflammatory cells and fibroblasts and recent evidence also suggests that the provisional ECM is a key regulator of EMT [8]. Moreover, fibrin inhibits surfactant function, resulting in alveolar collapse (atelectasis) [21]. This is potentiated by the release of fibrogenic growth factors from the fibrin matrix in a process known as collapse, termed "collapse induration". This, combined with traction of the remaining airspaces in the lung, results in the typical morphological pattern of fibrosis and honeycombing [22].

Using different in vivo models of bleomycin-induced pulmonary fibrosis, a number of studies have shown that fibrin persistence appears to promote fibrosis following inflammatory lung injury $[20,23]$. However, fibrinogen knock-out mice are not protected from bleomycin-induced fibrosis [24]. It is unclear whether these findings are a feature of these models or whether fibrin is not required for fibrosis to occur. Other factors might be more dominant in these models and, potentially, in patients. Interestingly, fibrin deposition is not commonly observed in patients with the characteristic histopathological appearance of usual interstitial pneumonia (UIP) but hyaline membrane formation and fibrin deposition have recently been reported to be common histopathological features of UIP patients with acute exacerbation, potentially as a result of diffuse alveolar damage [25]. Moreover, locally produced and activated coagulation zymogens can signal via their receptors in the absence of fibrin generation. Therefore, absence of fibrin does not necessarily imply the absence of the activation of coagulation factors and coagulation proteinase signalling.

\section{PROTEINASE-ACTIVATED RECEPTORS}

If fibrin is not required for fibrosis, how is the coagulation cascade involved in driving the fibrotic response? 


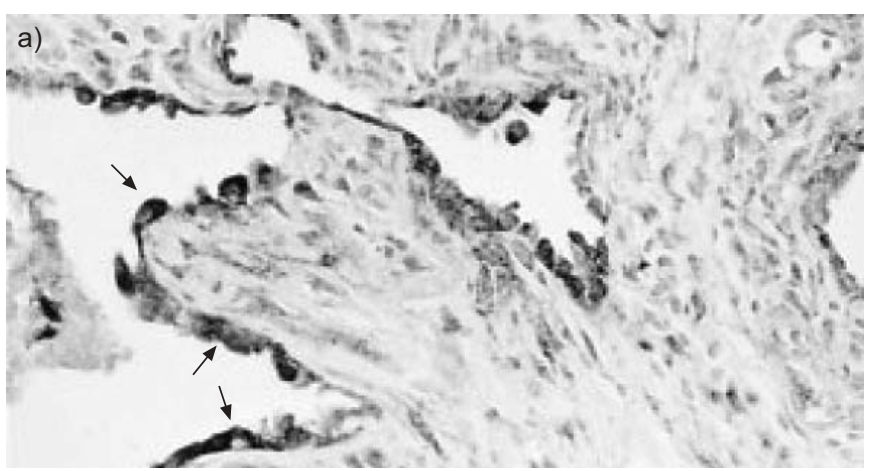

b) $\mathrm{TF} / \mathrm{FV} \mathrm{Ila}$

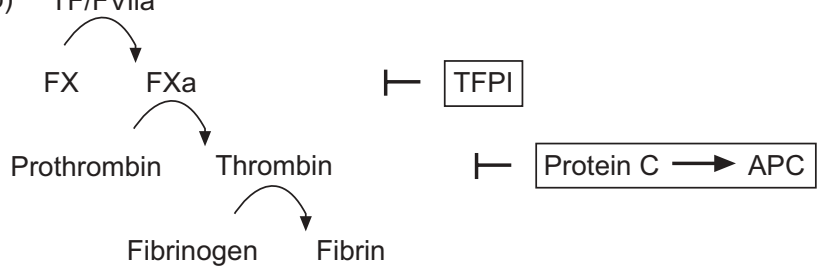

FIGURE 2. Tissue factor (TF)-dependent activation of the coagulation cascade in pulmonary fibrosis. a) TF immunoreactivity (arrows) associated with the epithelium overlying fibrotic foci in idiopathic pulmonary fibrosis. b) TF is the main initiator of the extrinsic coagulation pathway and binds and activates FVII. This leads to the activation of FX to FXa, which is then responsible for converting prothrombin to thrombin, the main effector proteinase of the coagulation cascade, which then converts soluble fibrinogen to insoluble fibrin. The extrinsic coagulation pathway is controlled by several endogenous anticoagulants, including TF pathway inhibitor (TFPI) and the protein C/activated protein C (APC) systems. Modified from [11] with permission from the publisher

The discovery of the proteinase-activated receptors (PARs) in the early 1990s [26] provided some answers to this question, in that these receptors provided a solution to the quandary as to how thrombin was able to mediate cellular responses and, in particular, promote platelet aggregation. The last $15 \mathrm{yrs}$ have seen major advances in the appreciation of the pluripotent cellular responses mediated via this novel family of signalling receptors, many of which may play crucial roles in influencing inflammatory and tissue repair responses following tissue injury.

The PAR family consists of four members (PAR1-PAR4) and belongs to a sub-family of the seven transmembrane domain $G$ protein-coupled receptors that signal in response to extracellular proteinases, including the major coagulation proteinases of the extrinsic pathway [27, 28]. The name of this receptor family derives from its unique mechanism of action, which involves the unmasking of a tethered ligand following limited proteolysis of $\sim 40 \mathrm{~N}$-terminal amino acid residues (fig. 3). The tethered ligand subsequently binds to the second extracellular loop of the seven transmembrane domain receptor [30] to initiate a conformational change at the $C$ terminus, and signalling via the recruitment of heterotrimeric G-proteins [31-33].

Collectively, the proteinases in the coagulation cascade can target all four PARs but these receptors can also be activated by noncoagulation proteinases. For example, PAR2 is a major substrate for trypsin and matrix metalloprotein (MMP)-1 has recently been reported to promote tumour growth and invasion through activation of PAR1 [34]. PAR1, PAR2 and PAR4 act as signalling receptors, but PAR3 appears to act as a thrombin docking receptor, binding and localising thrombin for efficient activation of PAR4 at low thrombin concentrations [35]. In addition, in human endothelial cells, the tethered ligand domain of cleaved PAR1 can signal by transactivating PAR2 [36], so that thrombin can in fact signal via all four PARs, although current evidence suggests that most of its cellular responses are mediated via its high-affinity receptor PAR1. This evidence in the context of fibrotic responses will now be reviewed.

\section{PAR1: A NOVEL TARGET IN IPF?}

PAR1 was the first PAR to be cloned and fully characterised and is expressed on numerous cell types that are resident in the lung, including endothelial cells, vascular and airway smooth muscle cells, fibroblasts, bronchial and alveolar epithelial cells, fibroblasts/myofibroblasts and various inflammatory cells recruited following injury, such as monocytes/ macrophages and T-cells [29]. Although several proteinases have been reported to activate PAR1 in vitro, there is little doubt that thrombin is a major physiological and primary activator of PAR1 (median effective concentration $50 \mathrm{pM}$ ). Other PAR1 activators, (FXa, trypsin, granzyme A, etc.) are generally considered "secondary activating proteinases" which activate PAR1 at high concentrations [37].

The physiological functions elicited following PAR1 activation in the context of tissue injury and repair responses can be loosely grouped into five major categories; 1) haemostasis; 2) endothelial barrier function; 3) inflammatory cell recruitment; 4) mesenchymal cell and endothelial cell mitogenesis; and 5) fibrogenic responses (table 2).

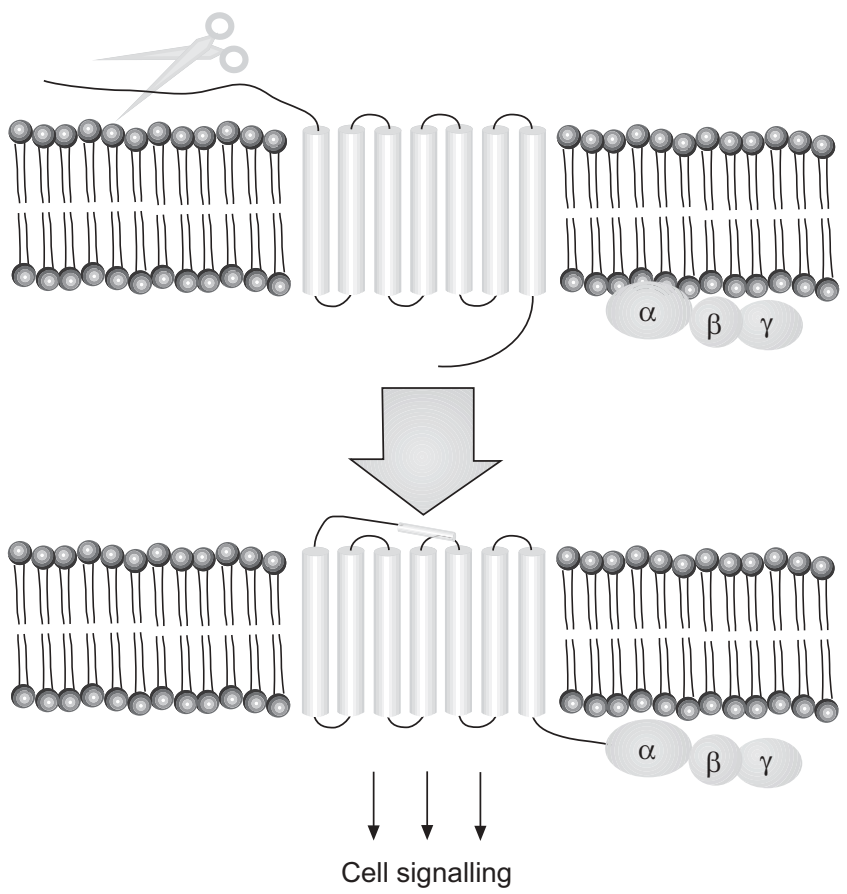

FIGURE 3. Activation of proteinase activated receptors. Reproduced from [29] with permission from the publisher 
As already mentioned, PAR1 is expressed on numerous cell types involved in promoting haemostasis and endothelial barrier function, including platelets, vascular smooth muscle cells and endothelial cells. Following damage to the vasculature, in addition to promoting platelet aggregation, activation of PAR1 promotes smooth muscle cell contraction to limit blood loss at sites of injury. PAR1 is the major thrombin receptor expressed on the microvascular endothelium and activation of PAR1 by thrombin plays a major role in regulating endothelial barrier function in a highly concentration dependent manner; low concentrations of thrombin $(<40 \mathrm{pM})$ mediate barrier protective responses while higher concentrations are barrier disruptive by promoting endothelial cell permeability and contraction [38]. Direct intravenous infusion of thrombin increases pulmonary vascular permeability in experimental models [39] and an important role for PAR1 in mediating these effects was provided by studies showing that thrombin-induced pulmonary microvascular permeability is abrogated in lung organ cultures from PAR1 knockout mice [40].

Activation of PAR1 on a variety of cell types, including epithelial cells, monocytes/macrophages and vascular endothelial cells, leads to the induction and release of potent pro-inflammatory mediators. These include: chemokines, such as interleukin (IL)-8 and CC motif chemokine ligand (CCL)2 (monocyte chemotactic protein-1); pro-inflammatory cytokines, such as tumour necrosis factor- $\alpha$, IL-1 $\beta$, IL-2 and IL-6; and the prostaglandin $\mathrm{E}_{2}$. Similar potent pro-inflammatory effects have also been reported for FXa and TF-FVIIa-FXa complexes, acting via both PAR1- and PAR2-dependent mechanisms, and there is increasing evidence that these PAR-mediated pro-inflammatory responses may play significant roles in the context of a number of conditions, including fibrosis [29, 41]. Moreover, thrombin has also been reported to

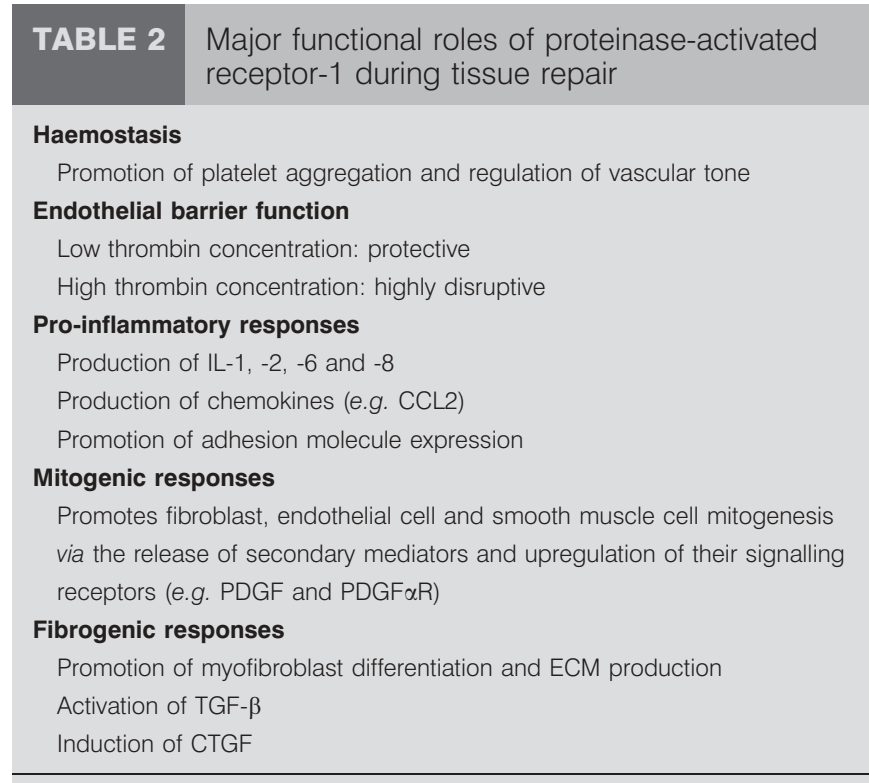

IL: interleukin; CCL2: CC-motif chemokine ligand 2; PDGF: platelet-derived growth factor; PDGFaR: PDGF $\alpha$ receptor; ECM: extracellular matrix; TGF: transforming growth factor; CTGF: connective tissue growth factor. induce the expression of endothelial cell adhesion molecules, including P-selectin and intercellular adhesion molecule-1 in vitro and may, therefore, facilitate the recruitment of inflammatory cells via both chemokine production and adhesion molecule expression [42].

Activation of PAR1 on numerous cell types, including smooth muscle cells, endothelial cells and fibroblasts initiates potent mitogenic responses via the induction and release of potent growth factors (e.g. PDGF). Whilst these responses might serve to initiate repair of damaged blood vessels and wound repair, if dysregulated, such responses may contribute to the development of tissue fibrosis by over-expanding the local resident fibroblast population at sites of injury.

Finally, activation of PAR1 on fibroblasts exerts potent fibrogenic responses by inducing fibroblast to myofibroblast differentiation [43] and promoting ECM protein production [44]. Current opinion suggests that some of these responses may be mediated via the induction of connective tissue growth factor (CTGF) [45], as well as the ability of PAR1 to influence $\alpha \mathrm{V} \beta 6$-dependent activation of TGF- $\beta$ on epithelial cells [46].

Evidence for the involvement of PAR1 in driving both inflammatory and fibrotic responses following lung injury was provided by studies in PAR1-deficient mice. These mice were found to be protected from both bleomycin-induced inflammatory cell recruitment and lung collagen accumulation. This protection was found to be associated with a reduction in the upregulation/activation of various PAR1-inducible mediators, including CCL2, CTGF and TGF- $\beta[46,47]$.

Demonstrating a causal role for PAR1 in the pathogenesis of pulmonary fibrosis, in particular IPF, is a challenging problem but the notion that this receptor may be involved is supported by immunohistochemical studies demonstrating that PAR1 is highly expressed on numerous cell types implicated in fibrosis, including macrophages and fibroblasts/myofibroblasts in the lungs of patients with IPF (fig. 4a) [47]. Moreover, PAR1 is also highly expressed in the lungs of patients with lung fibrosis associated with systemic sclerosis and is predominantly localised to fibroblasts/myofibroblasts present within early and late fibrotic lesions (fig. 4b) [43]. Recent unpublished data further suggests that the alveolar epithelium overlying fibrotic foci also expresses PAR1 and may represent a significant cellular source of the monocyte and fibrocyte chemoattractant, CCL2 (P.F. Mercer, University College London, London, UK; personal communication).

In terms of the proteinases involved in activating PAR1 in the context of fibrotic lung disease, current evidence from patient studies and animal models supports a major role for circulation-derived and locally activated thrombin [17, 48, 49]. However, it has been shown that PAR1 is also the major receptor by which FXa stimulates lung fibroblast procollagen production, proliferation and calcium signalling [50]. Current findings suggests that $\mathrm{FXa}$ can similarly induce fibroblast to myofibroblast differentiation (C.J. Scotton and M.A. Krupiczojc, University College London; personal communication). Studies examining whether direct FXa inhibition is protective in the bleomycin model are currently ongoing and should shed important light on the potential role of this proteinase in driving fibrotic responses in vivo. 

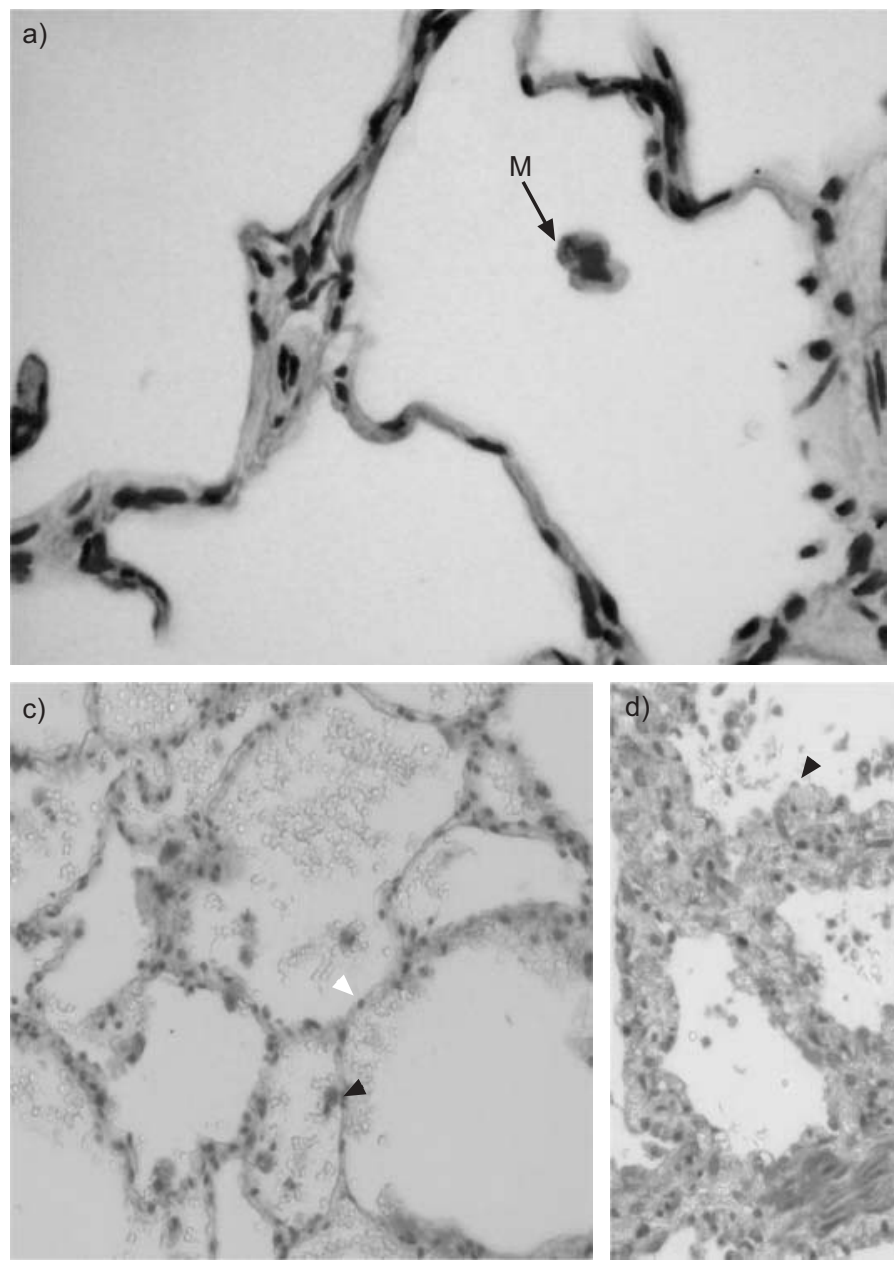

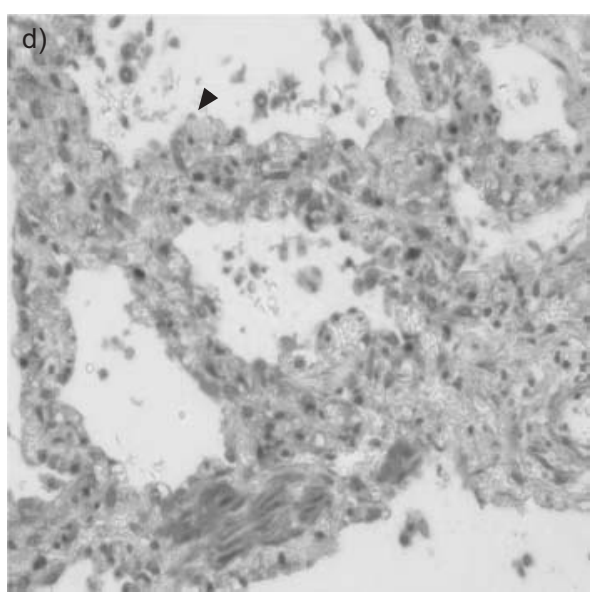

FIGURE 4. Proteinase activated receptor-1 expression in a) weak immunostaining in normal lung tissue, b) intense immunostaining in idiopathic pulmonary fibrosis, c) normal lung tissue, d) a patient with early-stage systemic sclerosis and e) a patient with late-stage systemic sclerosis. F: fibroblast; M: macrophage. Negatively and positively stained cells are represented by white and black arrowheads, respectively. Reproduced from [43] and [47] with permission from the publisher.

\section{IMPLICATIONS FOR THERAPY IN IPF}

Activation of the coagulation cascade is a feature of a number of lung disorders associated with excessive deposition of ECM, including IPF. Studies in experimental animal models have provided a compelling case that blocking the coagulation cascade and, more specifically, the pro-fibrotic effects of coagulation proteinases, may hold promise for the treatment of IPF. Moreover, a recent randomised controlled trial of anticoagulation and corticosteroids versus corticosteroids alone in 56 Japanese patients with IPF has highlighted the potential of anticoagulant therapy in this condition [51]. The anticoagulants included oral warfarin in an outpatient setting and low-molecular weight heparin for re-hospitalised patients with severely progressive respiratory failure. The results obtained suggest that plasma D-dimer levels (a surrogate marker for the activation of coagulation and fibrinolysis) are associated with mortality in patients with an acute exacerbation of IPF, and that anticoagulant therapy has a beneficial effect on survival in patients with this condition. However, these observations require confirmation by future studies addressing several concerns with the study design, including the nature of the patient cohort and the potential for misclassification bias, as well as dropouts affecting randomisation [52].

Initial data suggest that some of the coagulation pathways discussed in the present article may be important in idiopathic pulmonary fibrosis, especially in acute exacerbations. However, further studies to examine the role of coagulation pathways in patients with idiopathic pulmonary fibrosis are urgently required. From a clinical perspective, in terms of longterm anticoagulant therapy, it is now increasingly recognised that therapeutic approaches based on selective inhibitors of specific coagulation factors, such as FXa, rather than traditional, multi-targeted anticoagulants, such as warfarin and unfractionated heparin, are likely to be associated with a wider therapeutic window and would, therefore, be safer and easier to use [53]. Moreover, it has been argued for a while that inhibitors which selectively target the signalling properties of coagulation proteinases, without interfering with haemostatic responses, might be highly desirable in this patient group. The recent development of potent proteinase-activated receptor-1 antagonists may hold promise in this regard. Orally active 
proteinase-activated receptor- 1 antagonists are currently being evaluated in several phase II and phase III trials in the setting of cardiovascular disease [29]. The results of these trials are eagerly awaited and may hold promise for therapeutic intervention in the setting of a number of fibroproliferative conditions, including idiopathic pulmonary fibrosis.

\section{REFERENCES}

1 Horowitz JC, Thannickal VJ. Epithelial-mesenchymal interactions in pulmonary fibrosis. Semin Respir Crit Care Med 2006; 27: 600-612.

2 Scotton CJ, Chambers RC. Molecular targets in pulmonary fibrosis: the myofibroblast in focus. Chest 2007; 132: 1311-1321.

3 Hinz B, Phan SH, Thannickal VJ, Galli A, BochatonPiallat ML, Gabbiani G. The myofibroblast: one function, multiple origins. Am J Pathol 2007; 170: 1807-1816.

4 Abe R, Donnelly SC, Peng T, Bucala R, Metz CN. Peripheral blood fibrocytes: differentiation pathway and migration to wound sites. J Immunol 2001; 166: 7556-7562.

5 Phillips RJ, Burdick MD, Hong K, et al. Circulating fibrocytes traffic to the lungs in response to CXCL12 and mediate fibrosis. J Clin Invest 2004; 114: 438-446.

6 Andersson-Sjöland A, de Alba CG, Nihlberg K, et al. Fibrocytes are a potential source of lung fibroblasts in idiopathic pulmonary fibrosis. Int J Biochem Cell Biol 2008; 40: 2129-2140.

7 Willis BC, Borok Z. TGF- $\beta$-induced EMT: mechanisms and implications for fibrotic lung disease. Am J Physiol Lung Cell Mol Physiol 2007; 293: L525-L534.

8 Kim KK, Kugler MC, Wolters PJ, et al. Alveolar epithelial cell mesenchymal transition develops in vivo during pulmonary fibrosis and is regulated by the extracellular matrix. Proc Natl Acad Sci USA 2006; 103: 13180-13185.

9 Barth K, Reh J, Sturrock A, Kasper M. Epithelial versus myofibroblast differentiation in immortal rat lung cell lines-modulating effects of bleomycin. Histochem Cell Biol 2005; 124: 453-464.

10 Mann KG, Brummel K, Butenas S. What is all that thrombin for? J Thromb Haemost 2003; 1: 1504-1514.

11 Imokawa S, Sato A, Hayakawa H, Kotani M, Urano T, Takada A. Tissue factor expression and fibrin deposition in the lungs of patients with idiopathic pulmonary fibrosis and systemic sclerosis. Am J Respir Crit Care Med 1997; 156: 631-636.

12 Kotani I, Sato A, Hayakawa H, Urano T, Takada Y, Takada A. Increased procoagulant and antifibrinolytic activities in the lungs with idiopathic pulmonary fibrosis. Thromb Res 1995; 77: 493-504.

13 Magro CM, Waldman WJ, Knight DA, et al. Idiopathic pulmonary fibrosis related to endothelial injury and antiendothelial cell antibodies. Hum Immunol 2006; 67: 284-297.

14 Yasui H, Gabazza EC, Taguchi O, et al. Decreased protein $\mathrm{C}$ activation is associated with abnormal collagen turnover in the intraalveolar space of patients with interstitial lung disease. Clin Appl Thromb Hemost 2000; 6: 202-205.

15 Fujimoto H, Gabazza EC, Hataji O, et al. Thrombinactivatable fibrinolysis inhibitor and protein $\mathrm{C}$ inhibitor in interstitial lung disease. Am J Respir Crit Care Med 2003; 167: 1687-1694.

16 Kobayashi H, Gabazza EC, Taguchi O, et al. Protein C anticoagulant system in patients with interstitial lung disease. Am J Respir Crit Care Med 1998; 157: 1850-1854.

17 Howell DC, Goldsack NR, Marshall RP, et al. Direct thrombin inhibition reduces lung collagen, accumulation, and connective tissue growth factor mRNA levels in bleomycin-induced pulmonary fibrosis. Am J Pathol 2001; 159: 1383-1395.

18 Kijiyama N, Ueno H, Sugimoto I, et al. Intratracheal gene transfer of tissue factor pathway inhibitor attenuates pulmonary fibrosis. Biochem Biophys Res Commun 2006; 339: 1113-1119.

19 Yasui H, Gabazza EC, Tamaki S, et al. Intratracheal administration of activated protein $C$ inhibits bleomycininduced lung fibrosis in the mouse. Am J Respir Crit Care Med 2001; 163: 1660-1668.

20 Günther A, Lübke N, Ermert M, et al. Prevention of bleomycin-induced lung fibrosis by aerosolization of heparin or urokinase in rabbits. Am J Respir Crit Care Med 2003; 168: 1358-1365.

21 Seeger W, Elssner A, Günther A, Krämer HJ, Kalinowski HO. Lung surfactant phospholipids associate with polymerizing fibrin: loss of surface activity. Am J Respir Cell Mol Biol 1993; 9: 213-220.

22 Günther A, Ruppert C, Schmidt R, et al. Surfactant alteration and replacement in acute respiratory distress syndrome. Respir Res 2001; 2: 353-364.

23 Eitzman DT, McCoy RD, Zheng X, et al. Bleomycininduced pulmonary fibrosis in transgenic mice that either lack or overexpress the murine plasminogen activator inhibitor-1 gene. J Clin Invest 1996; 97: 232-237.

24 Hattori N, Degen JL, Sisson TH, et al. Bleomycin-induced pulmonary fibrosis in fibrinogen-null mice. Clin Invest 2000; 106: 1341-1350.

25 Parambil JG, Myers JL, Ryu JH. Histopathologic features and outcome of patients with acute exacerbation of idiopathic pulmonary fibrosis undergoing surgical lung biopsy. Chest 2005; 128: 3310-3315.

$26 \mathrm{Vu}$ TK, Hung DT, Wheaton VI, Coughlin SR. Molecular cloning of a functional thrombin receptor reveals a novel proteolytic mechanism of receptor activation. Cell 1991; 64: 1057-1068.

27 Macfarlane SR, Seatter MJ, Kanke T, Hunter GD, Plevin GD. Proteinase-activated receptors. Pharmacol Rev 2001; 53: 245-282.

28 Steinhoff M, Buddenkotte J, Shpacovitch V, et al. Proteinase-activated receptors: transducers of proteinasemediated signaling in inflammation and immune response. Endocr Rev 2005; 26: 1-43.

29 Chambers RC. Procoagulant signalling mechanisms in lung inflammation and fibrosis: novel opportunities for pharmacological intervention? Br J Pharmacol 2008; 153: Suppl. 1, S367-S378.

30 Coughlin SR. How the protease thrombin talks to cells. Proc Natl Acad Sci USA 1999; 96: 11023-11027.

31 Coughlin SR, Vu TK, Hung DT, Wheaton VI. Characterization of a functional thrombin receptor. Issues and opportunities. J Clin Invest 1992; 89: 351-355. 
32 Dery O, Corvera CU, Steinhoff M, Bunnett NW. Proteinase-activated receptors: novel mechanisms of signaling by serine proteases. Am J Physiol 1998; 274: C1429-C1452.

33 Hollenberg MD, Compton SJ. International Union of Pharmacology. XXVIII. Proteinase-activated receptors. Pharmacol Rev 2002; 54: 203-217.

34 Boire A, Covic L, Agarwal A, Jacques S, Sherifi S, Kuliopulos A. PAR1 is a matrix metalloprotease-1 receptor that promotes invasion and tumorigenesis of breast cancer cells. Cell 2005; 120: 303-313.

35 Nakanishi-Matsui M, Zheng Y-W, Weiss EJ, Sulciner D, Coughlin SR. PAR3 is a cofactor for PAR4 activation by thrombin. Nature 2000; 404: 609-613.

36 O'Brien PJ, Prevost N, Molino M, et al. Thrombin responses in human endothelial cells. Contributions from receptors other than PAR1 include the transactivation of PAR2 by thrombin-cleaved PAR1. J Biol Chem 2000; 275: 13502-13509.

37 Major CD, Santulli RJ, Derian CK, Andrade-Gordon P. Extracellular mediators in atherosclerosis and thrombosis: lessons from thrombin receptor knockout mice. Arterioscler Thromb Vasc Biol 2003; 23: 931-939.

38 Feistritzer C, Riewald M. Endothelial barrier protection by activated protein $\mathrm{C}$ through PAR1-dependent sphingosine 1-phosphate receptor-1 crossactivation. Blood 2005; 105: 3178-3184.

39 Siflinger-Birnboim A, Johnson A. Protein kinase C modulates pulmonary endothelial permeability: a paradigm for acute lung injury. Am J Physiol Lung Cell Mol Physiol 2003; 284: L435-L451.

40 Vogel SM, Gao X, Mehta D, et al. Abrogation of thrombininduced increase in pulmonary microvascular permeability in PAR-1 knockout mice. Physiol Genomics 2000; 4: 137-145.

41 Bunnett NW. Protease-activated receptors: how proteases signal to cells to cause inflammation and pain. Semin Thromb Hemost 2006; 32: Suppl. 1, 39-48.

42 Chambers RC. Proteinase-activated receptors and the pathophysiology of pulmonary fibrosis. Drug Dev Res 2003; 60: 29-35.
43 Bogatkevich GS, Gustilo E, Oates JC, et al. Distinct PKC isoforms mediate cell survival and DNA synthesis in thrombin-induced myofibroblasts. Am J Physiol Lung Cell Mol Physiol 2005; 288: L190-L201.

44 Chambers RC, Dabbagh K, McAnulty RJ, Gray AJ, BlancBrude OP, Laurent GJ. Thrombin stimulates fibroblast procollagen production via proteolytic activation of protease-activated receptor 1. Biochem J 1998; 333: 121-127.

45 Chambers RC, Leoni P, Blanc-Brude OP, Wembridge DE, Laurent GJ. Thrombin is a potent inducer of connective tissue growth factor production via proteolytic activation of protease-activated receptor-1. J Biol Chem 2000; 275: 35584-35591.

46 Jenkins RG, Su X, Su G, et al. Ligation of protease-activated receptor 1 enhances $\alpha \mathrm{V} \beta 6$ integrin-dependent TGF- $\beta$ activation and promotes acute lung injury. J Clin Invest 2006; 116: 1606-1614.

47 Howell DC, Johns RH, Lasky JA, et al. Absence of proteinase-activated receptor-1 signaling affords protection from bleomycin-induced lung inflammation and fibrosis. Am J Pathol 2005; 166: 1353-1365.

48 Hernandez Rodriguez NA, Cambrey AD, Harrison NK, Chambers RC, Gray AJ, Southcott AM. Role of thrombin in pulmonary fibrosis. Lancet 1995; 346: 1071-1073.

49 Ohba T, McDonald JK, Silver RM, Strange C, LeRoy EC, Ludwicka A. Scleroderma bronchoalveolar lavage fluid contains thrombin, a mediator of human lung fibroblast proliferation via induction of platelet-derived growth factor $\alpha$-receptor. Am J Respir Cell Mol Biol 1994; 10: 405-412.

50 Blanc-Brude OP, Archer F, Leoni P, et al. Factor Xa stimulates fibroblast procollagen production, proliferation, and calcium signaling via PAR1 activation. Exp Cell Res 2005; 304: 16-27.

51 Kubo H, Nakayama K, Yanai M, et al. Anticoagulant therapy for idiopathic pulmonary fibrosis. Chest 2005; 128: 1475-1482.

52 Kinder BW, Collard HR, King TE Jr. Anticoagulant therapy and idiopathic pulmonary fibrosis. Chest 2006; 130: 302-303.

53 Bauer KA. New anticoagulants: anti IIa versus anti Xa-is one better? J Thromb Thrombolysis 2006; 21: 67-72. 